
Verlag von VEI'I \& COMP. in Leipzig.

\section{NORDGERILANISCHES OBLIGATIONENRECHT.}

\section{Von}

Karl von Amira,

o. ö. Professor der Rechte an der Universităt München.

Erster Band. Altschwedisches Obligationenrecht.

gr. 8. 1882 . geh. $25 \%$.

Zweiter Band. Westnordisches Obligationenrecht. gr. 8. 1895 geh. 30

\section{DIE EHE IN ÄGYPTEN ZUR PTOLEMÄISCH-RÖMISCHEN ZEIT}

nach den griechischen Heiratskontrakten und verwandten Urkunden.

$$
\text { Von }
$$

Dr. iur. Johannes Nietzold.

gr. 8. 1903. geh. 3 460 Fु.

\section{FRAUEN-FABRIKARBEIT UND FRAUENFRAGE.}

Eine prinzipielle Antwort

auf die Frage der Ausschliessung der verheirateten Frauen aus der Fabrik.

Von

Dr. Ludwig Pohle,

Professor an der Handelsakademie zu Frankfurt a. M.

gr. 8. 1900. geh. 2 A6.

\section{DEUTSCHE}

\section{RECHTSQUELLEN DES MITTELALTERS.}

Gesammelt und herausgegeben

von

Herrm. Wasserschleben,

Doktor der Theologie und der Rechte, Gehelmrat.

gr. 8. 1892. geh. 8 H. 


\section{DIE \\ RECHTSSTELLUNG DER FRAU ALS GATTIN UND MUTTER}

GESCHICHTLICHE ENTWICKLUNG

IHRER PERSÖNLICHEN STELLUNG IM PRIVATRECHT

BIS IN DAS ACHTZEHNTE JAHRHUNDERT

VON

DR. ROBERT BARTSCH

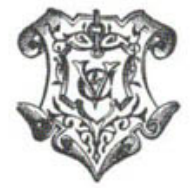

LEIPZIG

VERLAG VON VEIT \& COMP.

1903 
Verlag von VEI'T \& COMP. in Leipzig.

\title{
NORDGERIIANISCHES OBLIGATIONENRECHT.
}

\author{
Von
}

Karl von Amira,

o. ö. Professor der Rechte an der Universităt München.

Erster Band. Altschwedisches Obligationenrecht.

gr. 8. 1882 . geh. 25 16.

Zweiter Band. Westnordisches Obligationenrecht.

gr. 8. 1895 geh. 30 Æ.

\section{DIE EHE IN ÄGYPTEN}

ZUR PTOLEMÄISCH-RÖMISCHEN ZEIT

nach den griechischen Heiratskontrakten und verwandten Urkunden.

Von

Dr. iur. Johannes Nietzold.

gr. 8. 1903. geh. 3 A6 50 gg.

\section{FRAUEN-FABRIKARBEIT UND FRAUENFRAGE.}

Eine prinzipielle Antwort

auf die Frage der Ausschliessung der verheirateten Frauen aus der Fabrik.

$$
\text { Von }
$$

Dr. Ludwig Pohle,

Professor an der Handelsakademie zu Frankfurt a. $M$.

gr. 8. 1900. geh. 2 $\$ 6$.

DEUTSCHE

\section{RECHTSQUELLEN DES MITTELALTERS.}

Gesammelt und herausgegeben

von

Herrm, Wasserschleben,

Doktor der Theologie und der Rechte, Gehelmrat.

gr. 8. 1892. geh. 8 H. 


\section{DIE \\ RECHTSSTELLUNG DER FRAU ALS GATTIN UND MUTTER}

GESCHICHTLICHE ENTWICKLUNG

IHRER PERSÖNLICHEN STELLUNG IM PRIVATRECHT BIS IN DAS ACHTZEHNTE JAHRHUNDERT

VON

DR. ROBERT BARTSCH

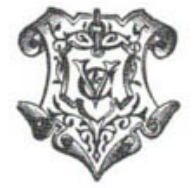

LEIPZIG

VERLAG VON VEIT \& COMP.

1903 
Druck von Metzger \& Wittig in Leipzig. 